\section{Original Article}

Korean J Transplant 2020;34:244-248

https://doi.org/10.4285/kjt.20.0053

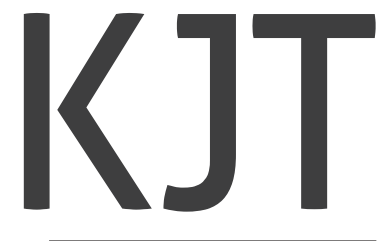

pISSN 2671-8790

elSSN 2671-8804

\title{
Serum creatinine level at 1-month posttransplant can independently predict long-term graft survival and functional status
}

\author{
Sung Min Koh, Man Ki Ju, Kyu Ha Huh, Yu Suen Kim, Myoung Soo Kim
}

Department of Surgery, Yonsei University College of Medicine, Seoul, Korea

Received November 13, 2020

Revised November 20, 2020

Accepted November 20, 2020

Corresponding author: Myoung Soo Kim Department of Surgery, Yonsei University College of Medicine, 50-1 Yonsei-ro, Seodaemun-gu, Seoul 03722, Korea Tel: +82-2-2019-3893

Fax: +82-2-3462-5994

E-mail:ysms91@yuhs.ac

Co-Corresponding author: Man Ki Ju Department of Surgery, Yonsei University College of Medicine, 50-1 Yonsei-ro, Seodaemun-gu, Seoul 03722, Korea Tel: +82-2-2019-3893

Fax: +82-2-3462-5994

E-mail:mkju@yuhs.ac

\footnotetext{
(c) The Korean Society for Transplantation This is an Open Access article distributed under the terms of the Creative Commons Attribution Non-Commercial License (http://creativecommons.org/licenses/ by-nc/4.0/) which permits unrestricted non-commercial use, distribution, and reproduction in any medium, provided the original work is properly cited.
}

Background: After the year 2000, kidney transplants with high immunologic risk and deceased donors increased rapidly in Korea. At the same time, the medical community developed special pretransplant and early posttransplantation management protocols. Our team evaluated the effect of early graft stabilization on long-term graft survival and functional status using databases from a high-volume kidney transplantation center. Methods: We included 1,895 kidney transplant patients from a total of 1,976 performed between 2005 and 2018. Early graft failure within 1 month $(n=9)$, loss to follow-up $(n=2)$, pediatric recipient or donor $(n=37)$, and combined organ transplantation $(n=33)$ cases were excluded. We grouped the cases at 1-month posttransplantation by serum creatinine quantiles (1.0 $\mathrm{mg} \%, 1.23 \mathrm{mg} \%$, and $1.52 \mathrm{mg} \%$ ).

Results: After an average of 95 months of follow-up (maximum 189 months), the high-serum creatinine group (4th quantile) showed significantly poorer graft survival than other groups (1st to 3rd quantile) $(\mathrm{P}<0.05)$. In multiple Cox regression analysis, a high serum creatinine level (4th quantile) at 1-month posttransplant is an independent risk factor for graft failure with a hazard ratio of $1.799(P=0.013)$. The quantile group by serum creatinine shows a persistent, significant difference of functional graft status (glomerular filtration rate by Modification of Diet in Renal Disease method) among quantile groups beyond ten years posttransplant.

Conclusions: Serum creatinine level at 1-month posttransplant is a strong independent predictor of graft survival and functional graft status beyond ten years posttransplant.

Keywords: Creatinine; Kidney transplant; Graft survival

\section{INTRODUCTION}

Kidney transplantation (KT) has improved patient survival and quality of life compared to other modes of renal replacement therapy. It has become the treatment of choice in end-stage renal failure [1]. However, the gradual decline in renal function after transplantation is inevitable [2]. The rate may be influenced by numerous variables, including both donor- and recipient-related characteristics. Donor age, donor and recipient race, cold ischemia time, human leukocyte antibody (HLA) mismatching, and delayed graft function have all been shown to significantly influence short- and long-term graft survival [3-5]. Moreover, a calcineurin inhibitor ( $\mathrm{CNI}$ ) based immunosuppression regimens exhibit dose-related nephrotoxicity and could be a contributing factor in graft function decline [6].

Early identification of those patients at risk of graft function and subsequent timely therapeutic intervention is 


\section{HIGHLIGHTS}

- One-month posttransplant serum creatinine level is related to long-term graft function.

- The glomerular filtration rate is associated with graft outcome.

- After the year 2000, higher risk kidney transplants increased rapidly in Korea.

necessary to improve outcomes in the kidney transplant patients.

Previous studies have shown that the parameters of renal function, mainly serum creatinine, creatinine clearance, and glomerular filtration rate (GFR), are valuable indicators of long-term outcomes. Early graft function, especially the 1 year posttransplant graft filtration rate or serum creatine level, is the most powerful predictor of long-term graft and patient survival [7-9]. However, these studies included patients who were treated in the early period of the transplantation era. Thus, the studies did not consider highly sensitized patients, $A B O$-incompatible transplantations, and marginal deceased donor.

After 2000, the number of kidney transplants of high immunologic risk patients (e.g., highly sensitized recipients, leucocyte cross-matching positive, and ABO-incompatible transplantation) and deceased donor transplantation, including marginal donors, rapidly increased in Korea. Special pretransplant and early posttransplantation management regimens were developed, such as plasmapheresis for antibody filtration and use of rituximab for antibody depletion [10]. At the same time, new immunosuppressive agents were rapidly developed $[11,12]$.

Given the recent changes, early graft stabilization after transplantation is essential. Therefore, we evaluated the effect of early graft stabilization on long-term graft survival and functional status using the databases from a large-volume KT center.

\section{METHODS}

We accessed the data of 1,976 consecutive KT recipients between 2005 and 2018 performed at Severance Hospital, Korea. We identified 1,895 cases that met our inclusion criteria. We excluded the following cases: graft failure within
1 month $(n=9)$, loss to follow-up $(n=2)$, pediatric recipient or donor (age under 18 years old, $n=37$ ), or combined organ transplantation $(n=33)$. We categorized the included patients into four groups defined by quartiles $(Q)$ of serum creatinine levels at 1-month posttransplant. The cut points for the groups were $1.0 \mathrm{mg} \%, 1.23 \mathrm{mg} \%$, and $1.52 \mathrm{mg} \%$. We used CNI based triple regimen (mycophenolate mofetil, steroid) as an immunosuppressants protocol, and after 6 months, steroid withdrawal is considered.

Our purpose of this study was comparing of graft survival of each group and comparing graft function and failure rate. Graft function was measured by estimated GFR by Modification of Diet in Renal Disease equation. Graft failure was defined as returning to dialysis or renal retransplantation. Categorical variables are presented as frequencies. We used the chi-square test or Fisher's exact test to evaluate these data. Time-to-event data were compared by Kaplan-Meier survival curves with log-rank tests. For the adjusted model, we used a Cox regression analysis. All analyses were performed using standard statistical software (IBM SPSS ver. 25.0; IBM Corp., Armonk, NY, USA) and $\mathrm{P}<0.05$ was considered statistically significant.

\section{RESULTS}

We had 450 individuals in the $1 \mathrm{st} \mathrm{Q}$ (serum creatinine $<1.0$ $\mathrm{mg} \%$ ), 476 in the $2 \mathrm{nd} \mathrm{Q}$ (serum creatinine $<1.23 \mathrm{mg} \%$ ), 489 in the $3 \mathrm{rd} \mathrm{Q}$ (serum creatinine $<1.52 \mathrm{mg} \%$ ), and 480

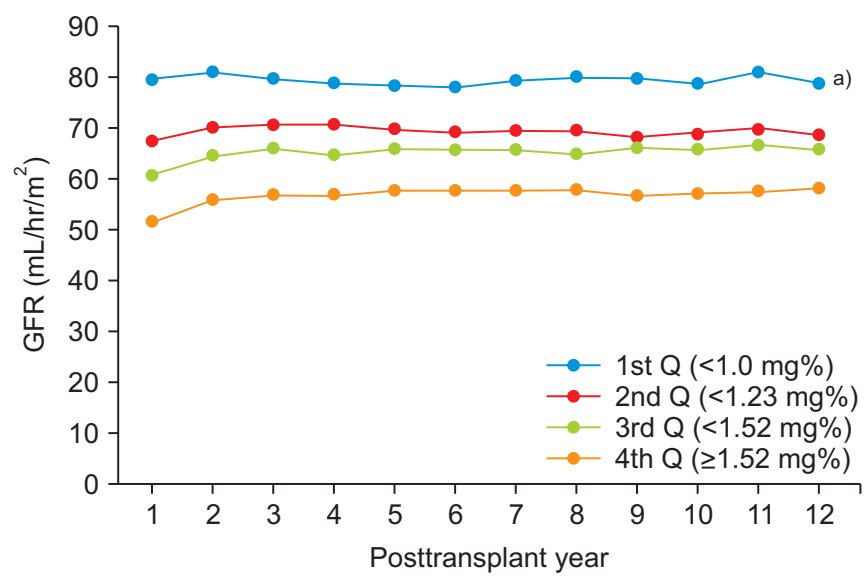

Fig. 1. Mean glomerular filtration rate (GFR) of functioning graft by quantile group of serum creatinine quantile at 1 -month posttransplant. Q, quartile. ${ }^{a)} \mathrm{P}<0.05$ vs. each other. 


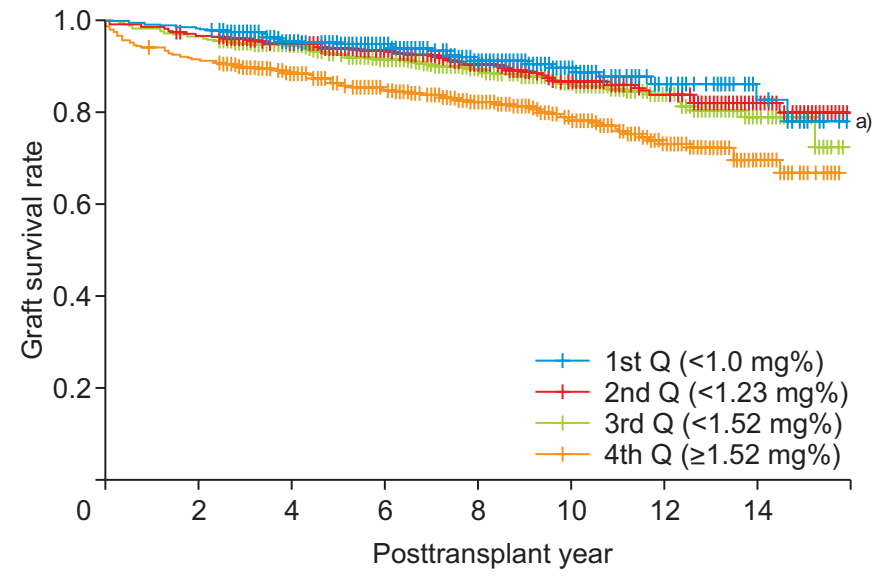

Fig. 2. Graft survival rate by quantile group of serum creatinine quantile at 1-month posttransplant. Q, quartile. ${ }^{\text {a) }} \mathrm{P}<0.05$ vs. others. in the 4th $\mathrm{Q}$ (serum creatinine $\geq 1.52 \mathrm{mg} \%$ ). With respect to high-risk transplantation, there were $582(30.7 \%)$ cases of ABO-incompatible transplants and $170(9.0 \%)$ cases of retransplantation, including four cases of three transplants and one case of four transplants. Finally, 286 (15.1\%) recipients underwent plasmapheresis at least one time (range, 1-7 times).

Serial changes of GFR for each group by year are shown in Fig. 1. The 1st Q group showed statistical significance compared to all other groups each year. Within the entire sample, there were $251 \mathrm{graft}$ failure events in the 1st Q group with 35 (8.8\%) cases, 2nd Q with 54 (11.3\%) cases, 3rd Q with 63 (12.9\%) case, and 4th Q with 99 (20.6\%) cases. The graft survival rate of the $1 \mathrm{st} Q$ was significantly higher than that of all the other groups at posttransplant

Table 1. Multiple Cox regression analysis for graft failure

\begin{tabular}{|c|c|c|c|c|}
\hline Variable & B & P-value & Hazard ratio & $95 \%$ Confidential interval \\
\hline Serum creatinine, posttransplant 1 month & & 0.023 & & \\
\hline 2nd Q (<1.23 mg\%) & 0.116 & 0.606 & 1.123 & $0.722-1.748$ \\
\hline 4th Q ( $\geq 1.52 \mathrm{mg} \%)$ & 0.585 & 0.013 & 1.795 & $1.133-2.843$ \\
\hline Retransplantation & 0.136 & 0.530 & 1.146 & $0.749-1.754$ \\
\hline Pretransplant desensitization & -0.264 & 0.461 & 0.768 & $0.382-1.547$ \\
\hline Recipient age (yr) & & 0.049 & & \\
\hline $25-34$ & -0.226 & 0.512 & 0.798 & $0.406-1.568$ \\
\hline $35-44$ & -0.567 & 0.101 & 0.567 & $0.288-1.117$ \\
\hline $45-54$ & -0.326 & 0.326 & 0.722 & $0.377-1.383$ \\
\hline $55-64$ & 0.002 & 0.996 & 1.002 & $0.515-1.950$ \\
\hline $35-44$ & -0.395 & 0.167 & 0.674 & $0.384-1.180$ \\
\hline $45-54$ & -0.146 & 0.586 & 0.864 & $0.511-1.462$ \\
\hline$\geq 55$ & 0.148 & 0.597 & 1.159 & $0.670-2.005$ \\
\hline Acute rejection within 1 year & 0.651 & 0.000 & 1.918 & $1.355-2.715$ \\
\hline HLA mismatching, zero-mismatchinga) & & 0.015 & & \\
\hline 1-3 Ag mismatching & 0.302 & 0.242 & 1.352 & $0.816-2.240$ \\
\hline 4-6 Ag mismatching & 0.665 & 0.014 & 1.944 & $1.146-3.298$ \\
\hline Donor sex, female & 0.323 & 0.016 & 1.382 & $1.063-1.796$ \\
\hline Recipient sex, female & 0.126 & 0.403 & 1.134 & $0.844-1.523$ \\
\hline Donor type, living related ${ }^{a}$ ) & & 0.000 & & \\
\hline
\end{tabular}

Q, quartile; HLA, human leukocyte antibody.

a) Reference value. 
every year (Fig. 2).

In the multivariate analysis, high serum creatinine level at 1-month posttransplant (4th quantile) is an independent risk factor for graft failure with a hazard ratio of $1.799(P=0.013)$. Posttransplant 1-month serum creatinine $(P=0.023)$, recipient age $(P=0.049), H L A$ zero-mismatching $(P=0.015)$, acute rejection within 1 year $(P<0.001)$, and a living related donor $(P<0.001)$ were shown to be statistically significant factors (Table 1).

\section{DISCUSSION}

This study showed that the 1-month posttransplant serum creatinine level is a strong predictor of long-term graft function for patients who had transplants after 2000. Moreover, the results reveal that some classical risk factors, such as HLA mismatching, donor type, and acute rejection within 1 year, affect long-term graft function. As the risk of early renal graft failure has substantially decreased over recent years, it is more critical to improve long-term graft function and the prevention of graft failure. It is also essential to find predictors of graft function to optimize the posttransplant care plan, including immunosuppressant combinations [13].

Previous studies have shown that 1-year posttransplant serum creatinine levels and GFR were strong predictive factors of long-term graft outcome [2-4]. However, these studies do not reflect the current state of KT as they were published one to two decades ago. There have been many changes in the transplantation field. High immunologic risk transplantation has been successfully performed, including $\mathrm{ABO}$-incompatible transplantation. The finding of this study that renal and graft failures was not influenced by advanced donor age was unexpected and is inconsistent with that of a previous report [14]. A study by De la Vega et al. [15] found that kidneys from living donors aged 50 years or older had a lower GFR pre- and posttransplant. Our study showed that only the recipient's age was related to graft failure.

This study is meaningful since it evaluated the longterm graft function of a substantial study population, including high-risk transplant patients $(752 / 1,895,39.6 \%)$. However, our study has some limitations. First, this is a retrospective single-institution study. The patient treatment protocol, including the immunosuppressive drug regimen, has changed during the study period. In summary, the 1-month posttransplant serum creatinine level is the most relevant parameter in predicting long-term graft function. It is influenced by recipient age, acute rejection, and donor type.

\section{ACKNOWLEDGMENTS}

\section{Conflict of Interest}

No potential conflict of interest relevant to this article was reported.

\section{ORCID}

Sung Min Koh https://orcid.org/0000-0002-7994-9977

Man Ki Ju https://orcid.org/0000-0002-4112-7003

Kyu Ha Huh https://orcid.org/0000-0003-1364-6989

Yu Suen Kim https://orcid.org/0000-0002-5105-1567

Myoung Soo Kim https://orcid.org/0000-0002-8975-8381

\section{Author Contributions}

Conceptualization: MKJ, MSK. Data curation: SMK. Methodology: MKJ, MSK. Formal analysis: MKJ, MSK. Investigation: MKJ, KHH, YSK. Project administration: MSK. Visualization: MKJ, MSK. Writing-original draft: SMK, MKJ. Writing-review \& editing: all authors.

\section{REFERENCES}

1. Wolfe RA, Ashby VB, Milford EL, Ojo AO, Ettenger RE, Agodoa $\mathrm{LY}$, et al. Comparison of mortality in all patients on dialysis, patients on dialysis awaiting transplantation, and recipients of a first cadaveric transplant. $\mathrm{N}$ Engl J Med 1999;341:1725-30.

2. Marcén R, Morales JM, Fernández-Rodriguez A, Capdevila L, Pallardó L, Plaza JJ, et al. Long-term graft function changes in kidney transplant recipients. NDT Plus 2010;3(Suppl_2):ii2-8.

3. Salahudeen AK, May W. Cold ischemia time: Its reduction and influence on renal allograft and recipient survival in the US over the last decade. Transplantation 2006;82:89.

4. Cecka JM. The OPTN/UNOS renal transplant registry. Clin Transpl 2004:1-16.

5. Held PJ, Kahan BD, Hunsicker LG, Liska D, Wolfe RA, Port FK, et al. The impact of HLA mismatches on the 
survival of first cadaveric kidney transplants. $\mathrm{N}$ Engl J Med 1994;331:765-70.

6. Naesens M, Kuypers DR, Sarwal M. Calcineurin inhibitor nephrotoxicity. Clin J Am Soc Nephrol 2009;4:481508.

7. Pascual $\mathrm{M}$, Theruvath $\mathrm{T}$, Kawai T, Tolkoff-Rubin N, Cosi$\mathrm{mi} A \mathrm{~B}$. Strategies to improve long-term outcomes after renal transplantation. N Engl J Med 2002;346:580-90.

8. Mayer AD, Dmitrewski J, Squifflet JP, Besse T, Grabensee $B$, Klein $B$, et al. Multicenter randomized trial comparing tacrolimus (FK506) and cyclosporine in the prevention of renal allograft rejection: a report of the European Tacrolimus Multicenter Renal Study Group. Transplantation 1997;64:436-43.

9. Margreiter R; European Tacrolimus vs Ciclosporin Microemulsion Renal Transplantation Study Group. Efficacy and safety of tacrolimus compared with ciclosporin microemulsion in renal transplantation: a randomised multicentre study. Lancet 2002;359:741-6.

10. Okada D, Okumi M, Kakuta Y, Unagami K, lizuka J, Takagi T, et al. Outcome of the risk-stratified desensitiza- tion protocol in donor-specific antibody-positive living kidney transplant recipients: a retrospective study. Transpl Int 2018;31:1008-17.

11. Chapman JR. Clinical renal transplantation: where are we now, what are our key challenges? Transplant Proc 2010;42(9 Suppl):S3-6.

12. Geissler EK, Schlitt HJ. The potential benefits of rapamycin on renal function, tolerance, fibrosis, and malignancy following transplantation. Kidney Int 2010;78:1075-9.

13. Chapman JR. Progress in transplantation: will it be achieved in big steps or by marginal gains? Am J Kidney Dis 2017;69:287-95.

14. Cecka JM. The UNOS Scientific Renal Transplant Registry. Clin Transpl 1999:1-21.

15. De La Vega LS, Torres A, Bohorquez HE, Heimbach JK, Gloor JM, Schwab TR, et al. Patient and graft outcomes from older living kidney donors are similar to those from younger donors despite lower GFR. Kidney Int 2004;66:1654-61. 